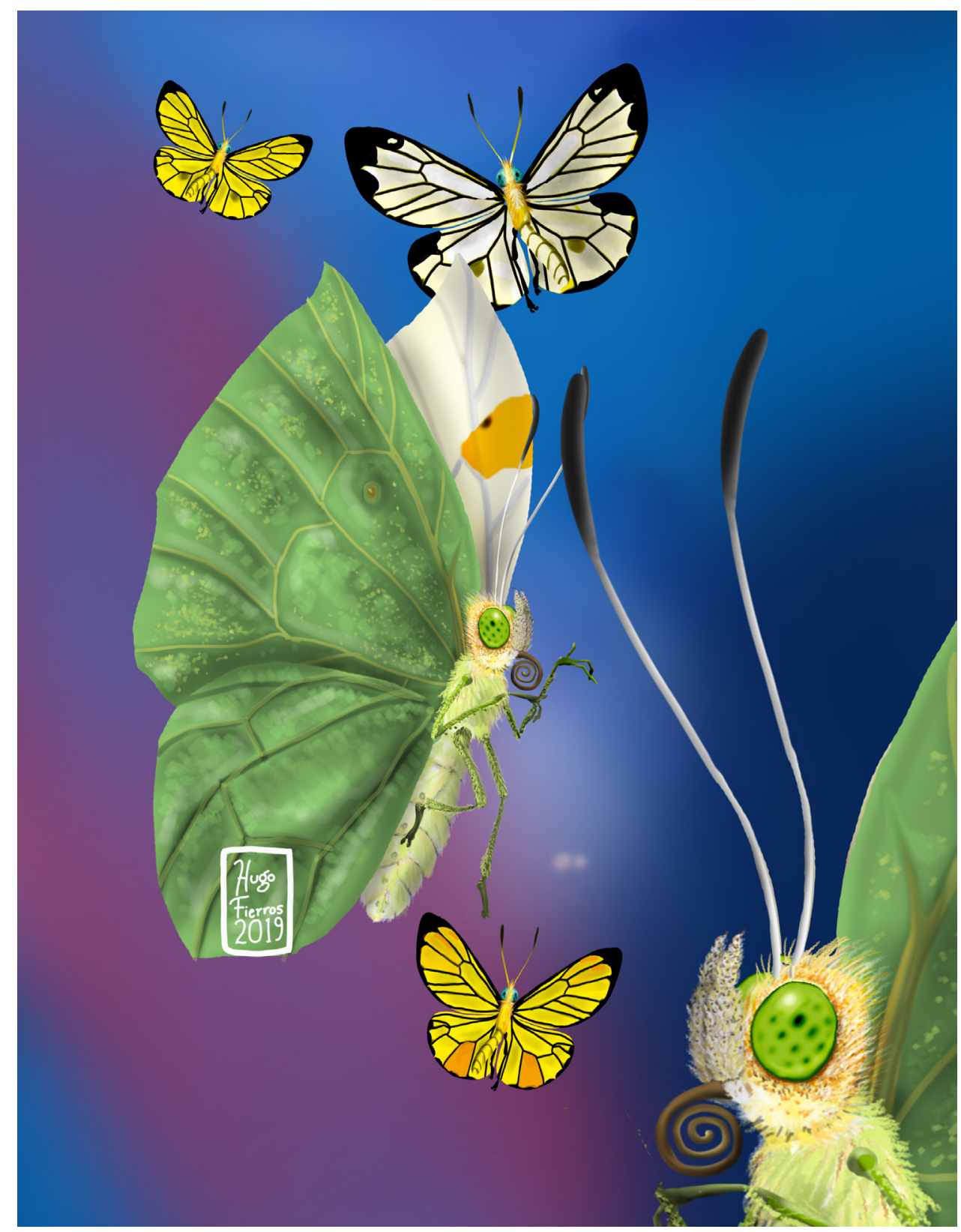

Dugesiana, Año 26, No. 2, julio 2019-diciembre 2019 (segundo semestre de 2019), es una publicación semestral, editada por la Universidad de Guadalajara, a través del Centro de Estudios en Zoología, por el Centro Universitario de Ciencias Biológicas y Agropecuarias. Camino Ramón Padilla Sánchez \# 2100, Nextipac, Zapopan, Jalisco, Tel. 37771150 ext. 33218, http://148.202.248.171/dugesiana/index.php/DUG/index, glenusmx@gmail.com. Editor responsable: José Luis Navarrete Heredia. Reserva de Derechos al Uso Exclusivo 04-2009-062310115100203, ISSN: 2007-9133, otorgados por el Instituto Nacional del Derecho de Autor. Responsable de la última actualización de este número: José Luis Navarrete Heredia, Editor y Ana Laura González-Hernández, Asistente Editorial. Fecha de la última modificación 25 de julio 2019, con un tiraje de un ejemplar.

Las opiniones expresadas por los autores no necesariamente reflejan la postura del editor de la publicación.

Queda estrictamente prohibida la reproducción total o parcial de los contenidos e imágenes de la publicación sin previa autorización de la Universidad de Guadalajara. 
Nota científica

\title{
Primer registro de pseudoescorpiones (Arachnida: Pseudoscorpiones) de Guanajuato, México
}

\author{
First record of pseudoscorpions (Arachnida: Pseudoscorpiones) from Guanajuato, México
}

\author{
Gabriel A. Villegas-Guzmán ${ }^{1,2}$ y Salvador Gaona ${ }^{1}$ \\ 'Departamente de Biología, Universidad Autónoma Metropolitana Iztapalapa, San Rafael Atlixco No. 186, Col. \\ Vicentina, C.P. 09340, Ciudad de México, México. ${ }^{2}$ Departamento de Zoología, Escuela Nacional de Ciencias \\ Biológicas, Instituto Politécnico Nacional, Prolongación Carpio y Plan de Ayala s/n, Col. Casco de Santo Tomás \\ C.P. 11340, Ciudad de México, México
}

Cuando se habla de biodiversidad en México generalmente se refiere a los vertebrados y las plantas, el país es considerado el segundo lugar a nivel mundial en diversidad de réptiles, el tercero en mamíferos, el cuarto en anfibios y el quinto en plantas (Semarnat 2013). Sin embargo, cuando se habla de artrópodos los datos son escasos, solo se conocen los datos de los insectos que hay en México de los cuales se tienen registrados a 47,853 especies (Semarnat 2013). En el caso de la clase Arachnida se han reportado para el país 5,752 especies (Francke 2014, Pérez et al 2014).

Los artrópodos son uno de los grupos más diversos en el mundo y dentro de éstos la clase Arachnida que comprende once órdenes, uno de ellos, Pseudoscorpiones, que de acuerdo con Harvey (2002) se considera un orden mesodiverso. En este encontramos 3,799 especies pertenecientes a 462 géneros y 27 familias (Harvey 2013). Para México se conocen 167 especies pertenecientes a 67 géneros y 18 familias (Villegas-Guzmán 2015). Los pseudoescorpiones se encuentran distribuidos en 29 estados de México; Colima, Tlaxcala y Guanajuato carecen de registros publicados (Ceballos 2004, Silva-Briano et al 2010), sin embargo hay datos de pseudoescorpiones de los primeros estados en el portal de los datos abiertos de la Colecciones Universitarias de la UNAM (https:// datosabiertos.unam.mx/biodiversidad). Es interesante notar que Colima y Tlaxcala son los estados más pequeños de México pero al mismo tiempo, históricamente poco explorados.

Guanajuato se ubica en el centro de México y presenta cuatro regiones ecogeográficas: Sierra Gorda, en el noreste del estado, las Sierras y Altiplanicie de la mesa central Guanajuatense, El Bajío que es una planicie en la Mesa Central, y las Sierras Volcánicas del sureste Guanajuatense (Cuevas-Carrillo y Zorrilla 2012), conformando un paisaje accidentado y diverso, a causa de esto existe una variedad de ecosistemas, $y$, por ende, una diversidad notable en flora y fauna. Es interesante resaltar que a pesar de esta diversidad fisiográfica, existen registros de 502 especies de artrópodos de dos clases y cuatro órdenes, la clase Insecta: Coleoptera (269), Lepidoptera (119), y Ortoptera (68), y la clase Arachnida: arañas (46) (Cruz Angún et al. 2012). Además, hay registros de nueve especies Scorpiones (PonceSaavedra y Francke 2013); se conocen cuatro especies de Opiliones para Guanajuato (Kury y Cokendolpher 2000) y en la Colección Nacional de Arácnido se han registrado a 41 ejemplares de Opiliones para este estado (MontielParra et al 2012). Lo anterior nos muestra lo poco que se ha estudiado en Guanajuato este phyllum.

Se recolectaron pseudoescorpiones en tres localidades, dos de San José Iturbide, La Tinaja (23 marzo 2017) en un bosque de encino uno en una trampa pit-fall $\left(20^{\circ} 57^{\prime} 23.8^{\prime \prime}\right.$ $\mathrm{N}, 100^{\circ} 22^{\prime} 3.4^{\prime \prime} \mathrm{W} 2,869 \mathrm{~m}$ ) y el otro en la misma localidad (5 abril 2017) pero en una necrotrampa $\left(20^{\circ} 57^{\prime} 14.3\right.$ ' N, $100^{\circ} 21^{\prime} 45.2$ " W, 2,901 m). Cuatro ejemplares de San Miguel de Allende, uno del Jardín Botánico "El Charco del Ingenio $\left(20^{\circ} 55^{\prime} 15.1\right.$ ' $\mathrm{N}, 100^{\circ} 43^{\prime} 39.6^{\prime}$ " W, 2,013 m) debajo de una roca (25 noviembre 2017), y tres ejemplares en un nido de Neotoma mexicana Baird, 1855 (22 febrero 2018) en el Rancho "El Estribo" (20 56 '25.1” N, 100 52' 14 "'W, $1,935 \mathrm{~m}$ ), ubicado a la altura del kilómetro $11+400$ de la carretera nueva a Guanajuato.

Los organismos se procesaron siguiendo la técnica descrita por Hoff (1949) con modificaciones de Wirth y Marston (1968). Los pseudoescorpiones se determinaron a especie, excepto uno de ellos que se hizo a nivel genérico debido a que era una tritoninfa y para conocer la especie requerimos observar caracteres que solo están presentes en los adultos. Los organismos se midieron de acuerdo a Chamberlin (1931) y a Benedict y Malcolm (1977). Los ejemplares están depositados en la Colección de Artrópodos Asociados a Mamíferos Silvestres de la Universidad Autónoma Metropolitana unidad Iztapalapa (CAAMSUAMI-149 al CAAMSUAMI-154).

Se encontraron seis pseudoescorpiones de tres familias, cuatro géneros y cuatro especies (Cuadro 1). Cuatro corresponden a la familia Chernetidae, tres de Parachernes ca robustus una hembra y dos deutoninfas en la cámara 
de reposo de un nido de Neotoma mexicana y uno de Hesperochernes sp., debajo de una roca. De las familias restantes cada una presentó un ejemplar, Neobisiidae, una hembra Microbisium parvulum (Banks), y de Garypinidae un macho de Serianus dolosus Hoff, 1956 (Cuadro1).

Asociados a nidos de Neotoma mexicana se han registrado a cinco especies de pseudoescorpiones pertenecientes a cuatro familias (Villegas-Guzmán y Pérez, 2005), de estas, dos son representantes de la familia Chernetidae: Illinichernes distinctus Hoff, y Tychochernes inflatus Hoff, por lo que este es el primer registro del género Parachernes asociado a nidos de $N$. mexicana, estableciendo una relación muy probablemente forética de acuerdo a lo mencionado por Muchmore (1971), quien indica que los pseudoescorpiones que se encuentran en los nidos de pequeños mamíferos o sobre ellos son foréticos. Los ejemplares encontrados consideramos que son cercanos a $P$. robustus, debido a que coinciden en algunas características morfológicas como: el tamaño y la presencia de una quilla en el carapacho (Fig. 1A), el tamaño de la tibia del pedipalpo (Fig. 1B), el número de segmentos que conforman las patas (Fig. 1C) y el número de dientes contiguoa de los dedos de la quela (Fig. 1D). Sin embargo, el ejemplar aquí reportado presenta muchas diferencias en tamaño de estructuras como: tamaño del cuerpo, quelícero, segmentos del pedipalpo y los segmentos de las patas I y IV. No podemos decir que se trata de una nueva especie, porque la descripción de $P$. robustus la hizo Hoff (1946) considerando únicamente a un ejemplar macho y el nuestro, es una hembra, por lo cual debemos encontrar al macho para compararlo adecuadamente y concluir si pertenece a esta especie o no. De este pseudoescorpión se encontró el estadio de deutoninfa, lo que indica que están realizando todo su ciclo de vida en este microhábitat.

El ejemplar determinado como Hesperochernes coincide con la mayoría de las características de este género: la relación largo y ancho del fémur y patella del pedipalpo (Fig. $2 \mathrm{~A}$ ), el número los dientes del dedo móvil y fijo de la quela (Fig. 2B). Sin embargo, no se puede determinar a nivel de especie, porque es una tritoninfa y no se observan los caracteres que definen a la especie, como: la espermateca terminalmente expandida, las sedas de la genitalia y la mano del quelícero con las sedas $a$ y $s b$ denticuladas.

Con respecto a Serianus dolosus, nuestro ejemplar es muy parecido a la descripción realizada por Hoff (1956) debido a la presencia de dos pares de ojos en el carapacho (Fig. 3A), el numero de segmentos de las patas y la seda de las genitalia (Fig.3 C). Aunque presenta menor tamaño del cuerpo (2.07 vs 2.2), la relación largo/ancho del fémur (3.0 vs 2.9 ) y la patella (2.6 vs 2.4) del palpo, son ligeramente mayores (Fig. 3B), el largo de la quela que son parecidas (0.647 vs 0.65 ) y comparten el mismo número de dientes contiguos en ambos dedos de la quelas con 20 (Fig. 3D). En relación con su hábitat, esta especie ha sido encontrada debajo de rocas y en hojarasca de encinos (Hoff 1956), por lo cual no es extraño haberla encontrado en una trampa pit fall en un bosque de encino.

En el caso de Microbisium parvalum, unas de las características morfológicas que se han usado para distinguirla son: el largo del fémur del palpo y la proporción largo/ancho de esta estructura (Nelson 1984). Nuestro ejemplar coincide con las medidas obtenidas por Nelson (1984) para ejemplares recolectados en Nuevo León y Tamaulipas, así como con las demás características asociadas a la especie; entre las que destacan: el tamaño del cuerpo, la quetotaxia de tergitos y esternitos (Fig. 4A), características del pedipalpo (Fig. 4B), el número de dientes de los dedos de la quela y las forma de las patas (Fig. 4C).

Con los datos obtenidos damos conocer los primeros registros de pseudoescorpiones para el estado de Guanajuato, además se establecen también por vez primera la posible relación simbiótica entre el género Parachernes en nidos de la rata montera Neotoma mexicana.

\section{AGRADECIMIENTOS}

A las autoridades del Jardín Botánico "El Charco del Ingenio" y al señor Vicente Yasbek por permitirnos recolectar en sus instalaciones. A Esteban Jiménez por el material de pseudoescorpiones de las trampas trapas de pitfall proporcionados. AÓscar Francke por sus comentarios y sugerencias al manuscrito. A PRODEP por el apoyo al proyecto: Ectoparásitos asociados a roedores de las familias Cricetidae y Heteromyidae del municipio de San Miguel de Allende, Guanajuato. Este trabajo se dedica al Dr. Jorge Llorente Bousquets en reconocimiento a sus contribuciones a la entomología mexicana.

\section{LITERATURA CITADA}

Benedict, E. M. and D. R. Malcolm. 1977. Some garypoid false scorpions from western North America (Pseudoscorpionida: Garypidae and Olpiidae). The Journal of Arachnology; 5 (2):113-132.

Chamberlin, J. C. 1931. The arachnid order Chelonethida. Stanford University Publications, Biological Sciences; 7(1):1-284.

Ceballos, A. 2004. Pseudoscorpionida, (pp. 417-429). In: J. E. Llorente-Bousquets, J. J. Morrone, O. Y. Ordóñez y I. V. Fernández (Eds.), Biodiversidad, taxonomía y biogeografía de Artrópodos de México: hacia una sintesis de su conocimiento. Vol. 4. Facultad de Ciencias, UNAM, CONABIO, México.

Cuevas-Carrillo, J. I. y M. Zorrilla. 2012. Localización y superficie. (pp. 28-37) In: La Biodiversidad en Guanajuato: Estudio de Estado vol. I. Conabio (Eds.). Comisión Nacional para el Conocimiento y Uso de la Biodiversidad (CONABIO) Instituto de Ecología del Estado de Guanajuato (IEE). Distrito Federal, México.

Cruz Angún, A., R. Becerril Patlán y $\mathrm{O}$. Báez Montes 2012. Introducción, (pp. 13-25). In Conabio (eds) la Biodiversidad en Guanajuato: Estudio de Estado vol. I. México. Comisión Nacional para el Conocimiento y Uso de la Biodiversidad (CONABIO) Instituto de 
Ecología del Estado de Guanajuato (IEE). Distrito Federal, México.

Francke, O. F. 2014. Diversidad de Arthropoda (Chelicerata: Arachnida ex Acari) en México. Revista Mexicana de Biodiversidad, Supl. 85:S408-S418.

Francke, O. F. and G. A.Villegas-Guzmán. 2006. Symbiotic relationships between pseudoscorpions (Arachnida) and packrats (Rodentia). Journal of Arachnology, 34, 289-298.

Harvey, M. 2002. The neglected cousins: What do we know about the smaller arachnid orders?. The Journal of Arachnology; 30(2):357-372.

Harvey, M. 2013. Pseudoscorpions of the World, version 3.0. Western Australian Museum Perth. Http://www. museum.wa.gov.au/catalogues/pseudoescorpions. Consultado 2 marzo 2018.

Hoff, C. C. 1946. New pseudoescorpions, chiefly Neotropical of the suborder Monosphyronida. American Museum Novitates; (1318):1-32.

Hoff, C.C. 1949. The pseudoscorpions of Illinois. Illinois Natural History Survey Buletin; 24, 409-498.

Hoff, C. C. 1956. Diplosphyronid pseudoscorpions from New Mexico. American Museum Novitates; (1780):149.

Kury, A. B. and J. C. Cokendolpher. 2000. Opiliones. (pp. 137-157). In: J. E. Llorente-Bousquets, E. GonzálezSoriano y J. J. Morrone (Eds.), Biodiversidad, taxonomía y biogeografía de Artrópodos de México: hacia una sintesis de su conocimiento. Vol. 3. Facultad de Ciencias, UNAM, CONABIO, México.

Montiel-Parra, G., D. R. Guerrero-Fuentes, J. A. CruzLópez y O. F. Francke. 2012. Estado actual del acervo de opiliones (Arachnida: Opiliones) en la Colección Nacional de Arácnidos del Instituto de Biología, UNAM. Entomología Mexicana, 11: 100-104.
Muchmore, W. B. 1971. Phoresy by North and Central American pseudoscorpions. Proceedings of the Rochester Academy Science, 12(2):77-97.

Nelson, S. Jr. 1984. The pseudoscorpion genus Microbisium in North and Central America (Pseudoecorpionida: Neobissidae). The Journal of Arachnology; 12(3):341350 .

Pérez, T. M., C. Guzmán-Cornejo, G. Montiel-Parra, R. Paredes-León y G. Rivas. 2014. Biodiversidad de ácaros en México. Revista Mexicana de Biodiversidad, Spl. 85: S399-S407.

Ponce Saavedra, J y O. F. Francke. 2013. Actualización taxonómica sobre los alacranes del Centro Occidente de México. Dugesiana, 20(2):73-79.

Silva-Briano, M., A. Adabache-Ortiz y G. GómezTorres. 2010. Introducción al conocimiento de los pseudoescorpiones del estado de Aguascalientes. Investigación y Ciencia de la Universidad Autónoma de Aguascalientes, 50:5-9.

Semarnat. 2013. Informe de la situación del medio ambiente en México. Compendio de estadística ambientales. Indicadores clave y de desempeño ambiental edición 2012. México.

Villegas-Guzmán, G. A: 2015. Pseudoscorpiones (Arachnida: Pseudoscorpiones) de la Ciudad de México y sus alrededores. Entomología mexicana, 2:76-81.

Villegas-Guzmán, G. A., y T. M. Pérez. 2005. Pseudoescorpiones (Arachnida: Pseudoscorpionida) asociados a nidos de ratas del género Neotoma (Mammalia: Rodentia) del Altiplano Mexicano. Acta Zoológica Mexicana (n. s.), 21, 63-77.

Wirth, W.W. and N. Marston. 1968. A method for mounting small insects on microscope slides in Canada balsam. Annals of the Entomological Society of America; 61(3):783-784.

Recibido: 4 de junio 2019

Aceptado: 19 de julio 2019 
Cuadro 1. Especies de pseudoescorpiones encontrados en dos municipios de Guanajuato. Donde T= tritoninfa y D= deutoninfa.

\begin{tabular}{|c|c|c|c|}
\hline Familia & Especie & Localidad & Habitat \\
\hline Neobidisiidae & Microbisium parvulum 1 우 & $\begin{array}{l}\text { La Tinaja } 20^{\circ} 57^{\prime} 14.3^{\prime \prime} \\
\mathrm{N}, 100^{\circ} 21^{\prime} 45.2^{\prime \prime} \mathrm{W}, \\
2,901 \mathrm{~m}\end{array}$ & $\begin{array}{l}\text { Bosque de Encino } \\
\text { (necrotrampa) }\end{array}$ \\
\hline Garypinidae & Serianus dolosus $1{ }^{\lambda}$ & $\begin{array}{l}\text { La Tinaja } 20^{\circ} 57^{\prime} 23.8^{\prime \prime} \\
\mathrm{N}, 100^{\circ} 22^{\prime} 3.4^{\prime \prime} \mathrm{W} \\
2,869 \mathrm{~m}\end{array}$ & $\begin{array}{l}\text { Bosque de Encino } \\
\text { (trampa pitfall) }\end{array}$ \\
\hline \multirow[t]{2}{*}{ Chernetidae } & Hesperochernes sp. 1T & $\begin{array}{l}\text { El Charco del } \\
\text { Ingenio20 } 50^{\circ}, 15.1{ }^{\prime}{ }^{\prime} \mathrm{N}, \\
100^{\circ} 43^{\prime} 39.66^{\prime \prime} \mathrm{W}, 2,013 \\
\mathrm{~m}\end{array}$ & Debajo de una roca \\
\hline & $\begin{array}{l}\text { Parachernes ca robustus } \\
1 ㅇ \text { y } 2 \text { D }\end{array}$ & $\begin{array}{l}\text { Rancho el estribo } 20^{\circ} \\
56^{\prime} 25.1^{\prime \prime} \mathrm{N}, 100^{\circ} 52^{\prime} \\
14^{\prime \prime} \mathrm{W}, 1,935 \mathrm{~m}\end{array}$ & $\begin{array}{l}\text { Nido de Neotoma } \\
\text { mexicana, cámara de } \\
\text { reposo }\end{array}$ \\
\hline
\end{tabular}
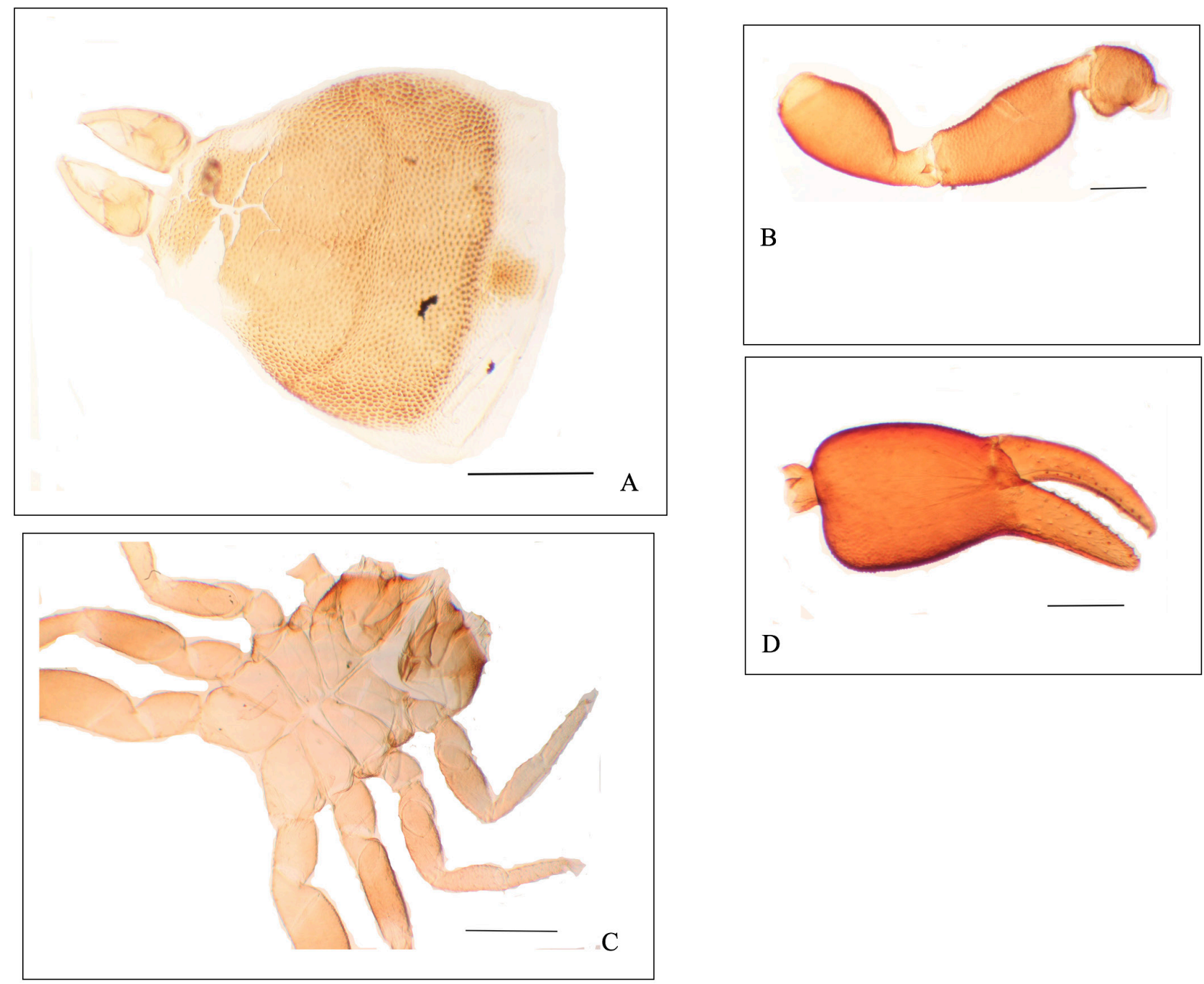

Figura 1. Partes de cuerpo de Parachernes ca robustus: (A) carapacho con quilla, (B) características del pedipalpo, (C) Número segmentos de las patas y (D) Dientes contiguos de los dedos de la quela. La línea representa en (A) $0.3 \mathrm{~mm}$, en (B), $0.1 \mathrm{~mm}$, en (C) 0.2 $\mathrm{mm}$ y en (D) $0.2 \mathrm{~mm}$. 

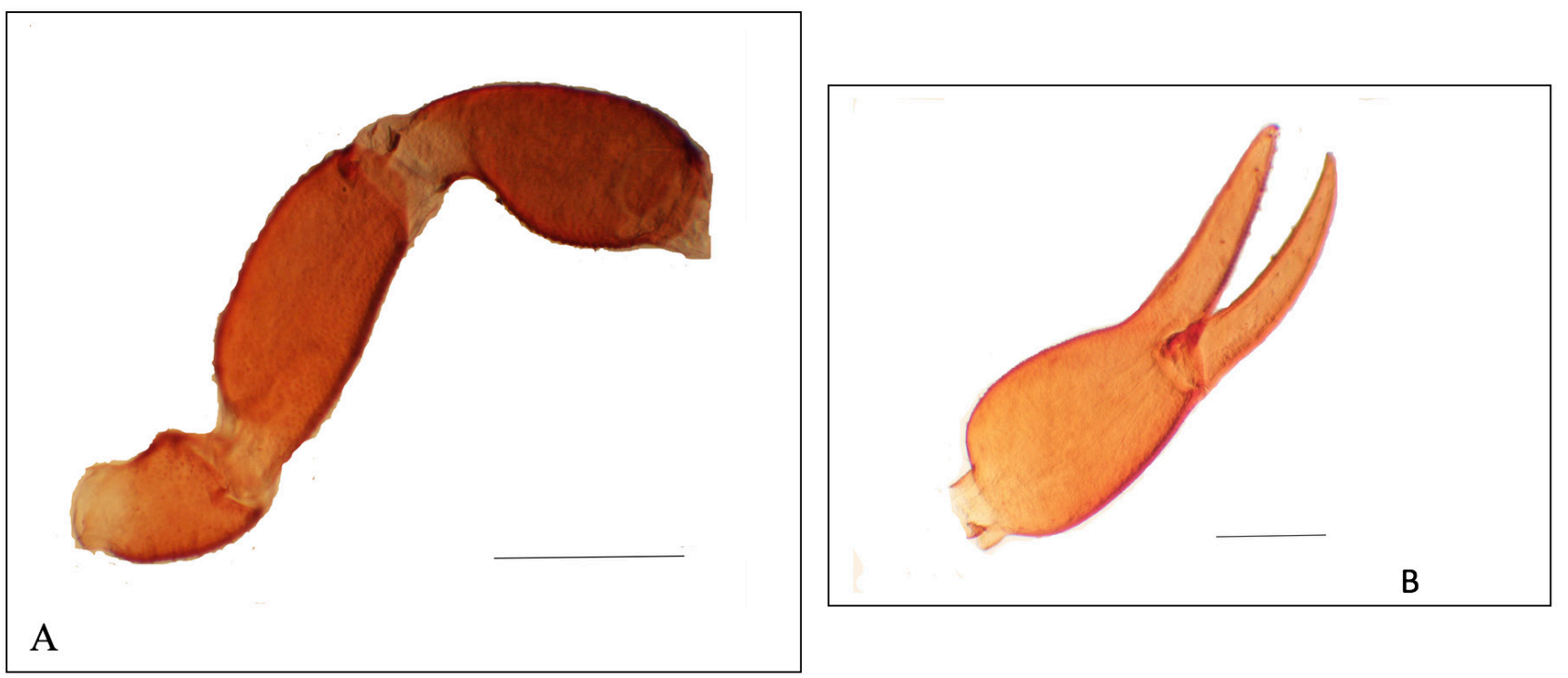

Figura 2. Hesperochernes sp., (A) características fémur y patella de pedipalpo, (B) dientes contiguos de los dedos quela. La línea la representa en A $0.4 \mathrm{~mm}$ y en B $0.2 \mathrm{~mm}$.
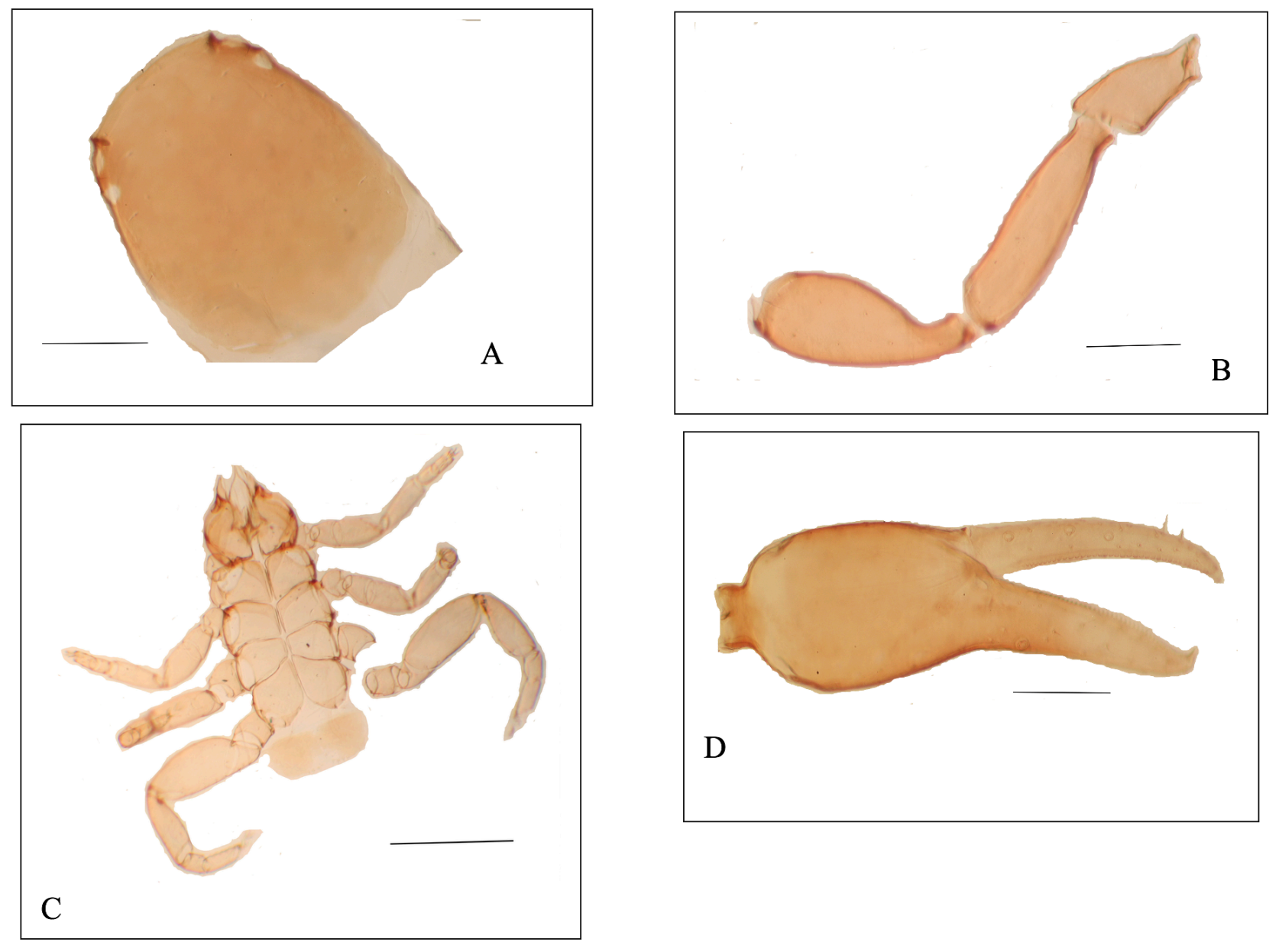

Figura 3. Partes de cuerpo de Serianus dolosus: (A) carapacho con dos pares de ojos, (B) características del pedipalpo, (C) número de segmentos de las patas y (D) dientes contiguos de los dedos de la quela. La línea representa en (A) $0.1 \mathrm{~mm}$, en (B), $0.2 \mathrm{~mm}$, en (C) 01 $\mathrm{mm}$ y en (D) $0.2 \mathrm{~mm}$. 

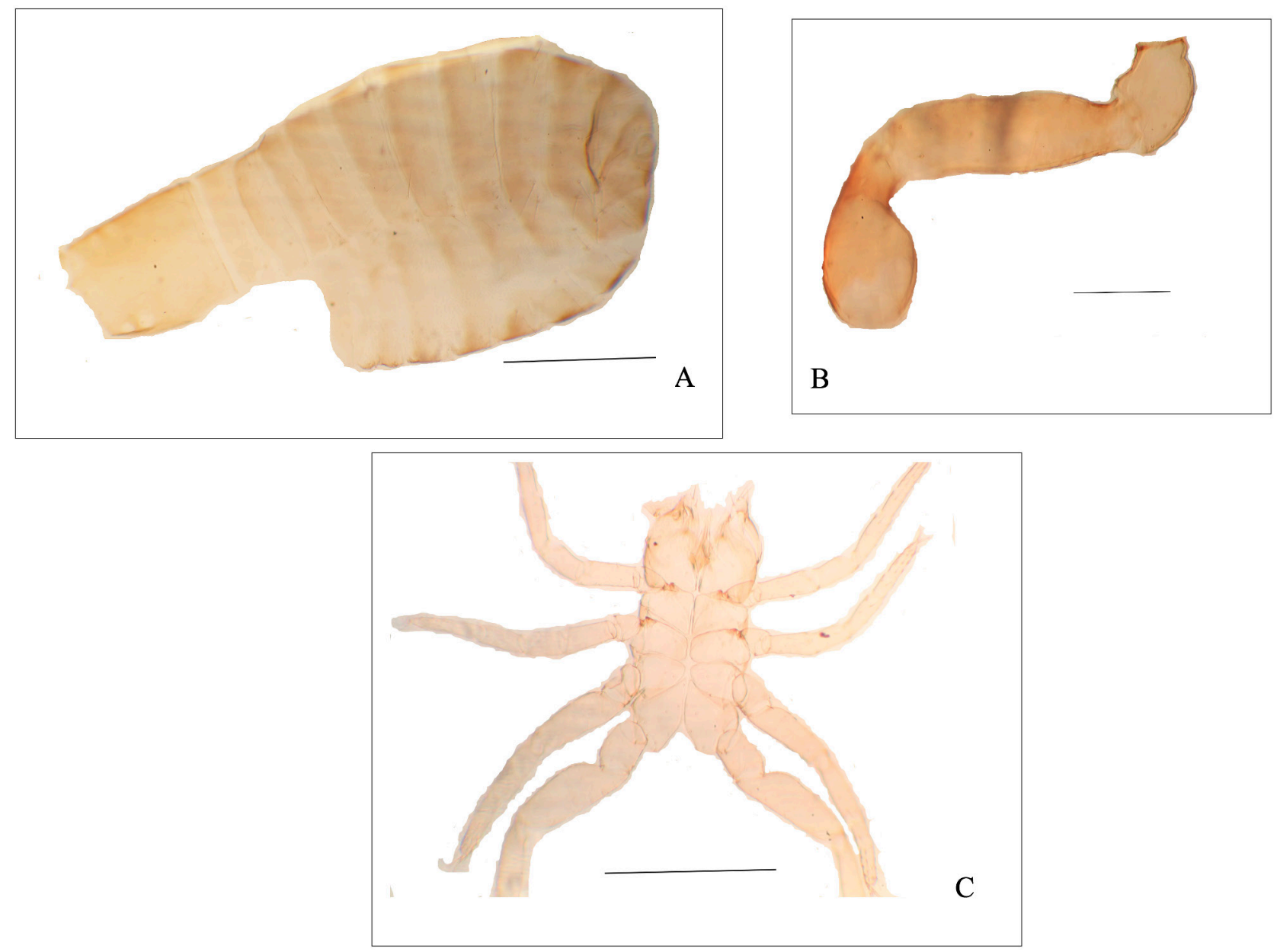

Figura 4. Partes de cuerpo de Microbisium parvalum: (A) cuerpo, tergitos y esternitos, (B) características del pedipalpo y (C) número de segmentos delas patas. La línea representa en (A) $0.5 \mathrm{~mm}$, en (B) y (C), $0.1 \mathrm{~mm}$. 